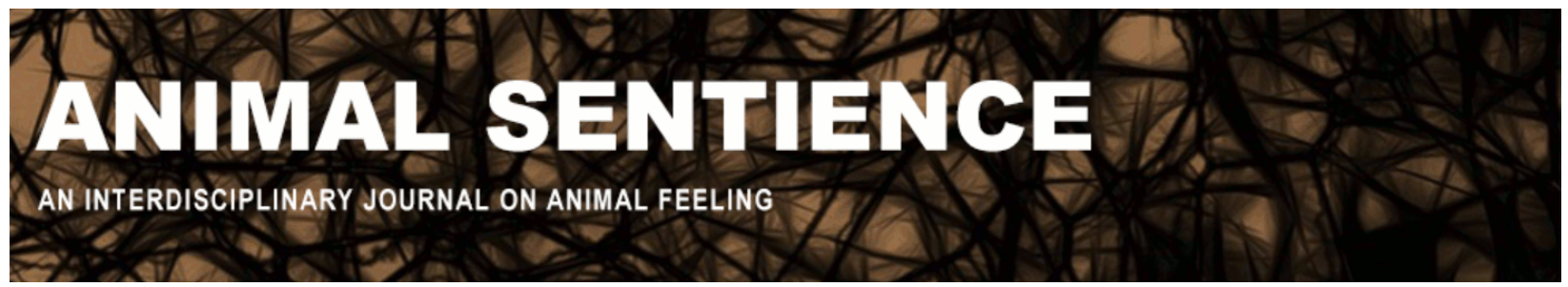

Woodruff, Michael L. (2016) Bacteria and the cellular basis of consciousness. Animal Sentience 11(2)

DOI: $10.51291 / 2377-7478.1152$

Date of submission: 2016-08-22

Date of acceptance: 2016-08-23

(c)

This article has appeared in the journal Animal

Sentience, a peer-reviewed journal on animal

cognition and feeling. It has been made open access,

free for all, by WellBeing International and deposited

in the WBI Studies Repository. For more information,

please contact

wbisr-info@wellbeingintl.org.

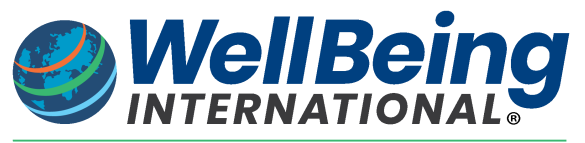

SOLUTIONS FOR PEOPLE, ANIMALS AND ENVIRONMENT 


\title{
Bacteria and the cellular basis of consciousness
}

Commentary on Reber on Origins of Mind

\author{
Michael L. Woodruff \\ Biomedical Sciences and Psychology \\ East Tennessee State University
}

\begin{abstract}
According to Reber's theory, the Cellular Basis of Consciousness (CBC), sentience originates as bio-sensitivity in unicellular organisms. For this reason, Reber regards sentience as evolutionarily foundational. Many bacteria show chemotaxis and, thus, according to CBC, they are sentient. Analysis of the genetic mechanisms underlying bacterial chemotaxis indicates that sentience has no explanatory power in this case. Genetic analysis also fails to show species continuity underlying bio-sensitivity in bacteria and bio-sensitivity in species with nervous systems, so it does not seem that sentience is evolutionary foundational. CBC is rejected on these grounds.
\end{abstract}

\begin{abstract}
Michael L. Woodruff is Professor Emeritus of Biomedical Sciences and of Psychology at East Tennessee State University. Author of more than 120 professional publications, his research interests include cognitive neuroscience and the philosophy of mind. https://www.researchgate.net/profile/Michael Woodruff3/info
\end{abstract}

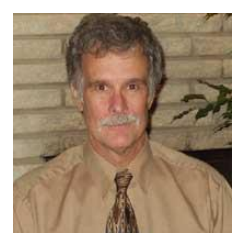

A basic postulate of Reber's (2016) Cellular Basis of Consciousness (CBC) theory is that "Any creature with flexible cell walls, a sensitivity to its surrounds and the capacity for locomotion..." (p. 4) is sentient. ${ }^{1}$ Thus, according to CBC theory, sentience begins in unicellular organisms that demonstrate what Reber calls "bio-sensitivity." Reber assigns evolutionary foundational status to sentience. That is, sentience might "become more complex and capable of greater behavioral and mental flexibility and power (p. 4)," but its fundamental subjective, experiential quality begins in unicellular species. This is an interesting argument, but it is unlikely to stand, as Reber would have it, "on evolutionary biological principles."

Many species of bacteria possess flagella containing filaments rotated by "molecular motors." The activity of the motors is driven by chemical signals from the environment mediated by receptors located on the surface of the bacterium. Stimulation of these receptors causes the motors to propel the bacterium toward a nutrient source or away from a toxin (Berg, 2003; Bren \& Eisenbach, 2000). This process is a specific example of Reber's "bio-sensitivity" and is called chemotaxis. Interestingly, when challenged by a nutrient-poor environment, bacteria experimentally deprived of the genes that build the flagella re-evolve these genes, and produce flagella (Taylor et al., 2015). Thus, chemotaxis has immense adaptive significance to bacteria. Bacterial chemotaxis can also be interpreted as causing goal-directed behavior, but is there any evidence that sentience is required to explain it?

\footnotetext{
1 Reber seems to use the terms mind, consciousness and sentience interchangeably to refer to subjective phenomenal states. For consistency I will use sentience and sentient where appropriate in referring to such states.
} 
According to $\mathrm{CBC}$ theory the answer to this question must be yes. While CBC theory mandates acceptance of reductive naturalism, sentience is not eliminated and remains in the ontology. If sentience is, then, a real thing and is also not epiphenomenal, it must have a function. The criteria for sentience provided by Reber requires that bacteria must be sentient. Thus, sentience must have a function in bacteria. Furthermore, since CDC asserts that sentience is reducible to biological mechanisms, it should be apparent in the mechanisms underlying chemotaxis in bacteria. Finally, if sentience is on an evolutionary continuum, even admitting "a gradual accretion of functions that are layered over and interlock with pre-existing ones (p. 5)," there must be some genomic continuity underlying bio-sensitivity in bacteria and bio-sensitivity in mammals.

Hox genes and their products may serve as an example of what I mean by genomic continuity. Hox genes are a group of related genes first found in Drosophila and subsequently found in all species examined to date, including humans. The number of Hox genes varies among phyla, but they always control the development of the structures associated with the segments of the body despite the vast differences in morphology of these structures across phyla and species (Shubin, 2008). If, as Reber maintains, sentience is evolutionarily foundational and shows species-continuity, something like Hox genes and their products should be apparent. Unfortunately for CBC theory this does not seem to be the case.

The genetic and molecular basis of bacterial chemotaxis has been well worked out. The details can be found in papers by Berg (2003) and Bren and Eisenbach (2000). I will provide only a cursory overview here. Several genes are responsible for the proteins that form the sensory receptor for chemotaxis. The mcp gene codes for the methyl-accepting chemotaxis protein (MCP). MCP spans the cell membrane and acts as the sensor protein. Two genes, cheW and cheA, code the proteins, CheW and CheA. These proteins are associated with the intracellular tails of MCP. CheW transduces the signal from MCP to CheA. CheA in turn activates the messenger protein $\mathrm{CheY}$ which migrates through the intracellular compartment to activate FliM, the switching protein that guides the direction of flagellar rotation. Some thirty genes are responsible for building the molecular motor. The principal genes associated with the stationary motor "housing," or stator, are mot $A$ and motB, while fliG, fliE, fliM, and fliN are primarily responsible for producing proteins associated with the motor's rotor.

So we have detailed knowledge concerning the genes behind the construction of the sensory and motor substrates underlying chemotaxis in bacteria. The biochemical basis of both the extracellular and intracellular transduction processes that cause the flagellum to rotate in one direction or the other are also understood. This information seems to be quite sufficient to explain bacterial chemotaxis without the introduction of sentience as an additional explanatory process. Furthermore, unlike Hox genes, the genes associated with the production of MCP, CheW and so on, do not demonstrate broad species continuity.

In sum, then, CBC theory fails at the level of two of its fundamental assumptions. First, there seems to be no empirical reason to set aside Occam's infamous razor and admit sentience as an explanatory variable to explain bacterial chemotaxis. Second, there is no good evidence that evolutionary continuity in the genetic substrates of bio-sensitivity exists between bacteria and organisms with nervous systems. Therefore, despite Reber's ambitious program, CBC does not supplant the more traditional view that a nervous system is necessary for sentience. Nor does it resolve the "hard problem." 


\section{References}

Berg, H. C. (2003). The Rotary Motor of Bacterial Flagella. Annual Review of Biochemistry, 72, 19-54.

Bren, A. \& Eisenbach, M. (2000). How Signals Are Heard during Bacterial Chemotaxis: ProteinProtein Interactions in Sensory Signal Propagation. Journal of Bacteriology, 182, 6865-6873.

Reber, A. S. (2016). Caterpillars, consciousness and the origins of mind. Animal Sentience 2016.106.

Shubin, N. (2008). Your Inner Fish: A Journey into the 3.5 Million Year History of the Human Body. New York: Pantheon.

Taylor, T. B., Mulley, G., Dills, A. H., Alsohim, A. S., McGuffin, L. J., Studholme, D. J., Silby, M. W., Brockhurst, M. A., Johnson, L. J. \& Jackson, R. W. (2015). Evolutionary resurrection of flagellar motility via rewiring of the nitrogen regulation system. Science, 347, 1014- 1017. 